\title{
A sequential contrast effect in odor perception
}

\author{
HARRY T. LAWLESS \\ Cornell University, Ithaca, New York
}

\begin{abstract}
An odor of ambiguous quality was shown to shift in rated odor character after presentation of more prototypical odors, always in a direction contrasting with the previous context. The terpene aroma compound, dihydromyrcenol, is perceived as partially woody (pine-like) and partially citrus (lime-like) in odor character. Citrus ratings of this odor increased following exposure to woody odors. Conversely, woody ratings of dihydromyrcenol increased following exposure to citrus odors. Possible explanations for this sequential contrast effect include shifts in cognitive category boundaries, response frequency biases, and simple sensory adaptation.
\end{abstract}

Contextual effects that produce effects of contrast or perception of enhanced differences are common in many sense modalities. Examples include color contrast, orientationcontingent color aftereffects, visual motion aftereffects, and adaptation to visual patterns of specific spatial frequencies (see, e.g., Blakemore \& Sutton, 1969). In the sense of taste, adaptation of the tongue to a solution of bitter caffeine will make a weak sucrose solution appear sweeter than it would after exposure to water (McBurney \& Bartoshuk, 1973), an effect attributed to the addition of water tastes. Speech sounds near phoneme boundaries shift to adjacent categories following adaptation, an effect that has given rise to controversies concerning the fatigue of specific feature detectors (Diehl, 1981; Eimas \& Corbit, 1973).

Although in olfactory cross-adaptation studies changes in smell intensity (see, e.g., Koster, 1971) have been measured following exposure to various odors, contrast effects on odor quality have received little attention. Part of the difficulty in measuring changes in odor quality arises from the lack of a generally accepted framework concerning how the qualitative attributes of odors should be classified, characterized, and measured (Engen, 1982). Recently, multidimensional scaling of citrus and woody terpene aroma materials showed that some ambiguous or boundary compounds fell between citrus and woody classes. These materials included compounds such as dihydromyrcenol, which are partly lime-like and partly pine-like in odor character. The multistable nature of dihydromyrcenol was corroborated in informal observations by an industrial sensory panel during training in fragrance evaluation. The panel was inspecting reference materials to help calibrate their use of odor attributes such as "woody" and "citrus." The panelists remarked that dihydromyrcenol seemed too woody to be given as an example of citrus-type fragrances.

Part of these results were presented at the 1988 meeting of the Psychonomic Society. The author thanks David A. Stevens and Terry Acree for thoughtful discussions. Reprint requests should be sent to Harry Lawless, Department of Food Science, Cornell University, Ithaca, NY 14853.
When this same odorant was later presented during a training session in which woody fragrance examples were being inspected, the same panelist/trainees then remarked that this odorant was far too citrus-like to serve as an example of a woody odor. The experiment outlined below provides quantitative documentation of this context-dependent shift in odor quality.

\section{METHOD}

Four groups of subjects were run on separate days. The groups consisted of $60,60,51$, and 59 adult females. These subjects were members of a panel of employee volunteers who routinely evaluated the acceptability of fragrances for use in consumer products and in stability (shelf-life) tests. Because of the routine nature of their participation in product tests, there was approximately an $80 \%$ overlap in the subjects constituting any two groups.

The main stimulus of interest was dihydromyrcenol, a terpene aroma compound with both lime-like and pine-like fragrance character. In one condition, dihydromyrcenol was evaluated following ratings of a "citrus" set of aroma materials, namely citral, lemon oil, orange oil, d-limonene, and mandarin oil. In a second condition, dihydromyrcenol was rated following a "woody" set of aroma materials, namely pine oil, cedrene, cedarwood oil, sandalwood oil, and isobornyl acetate. Previous multidimensional scaling had confirmed that these odorants were sorted by naive subjects into citrus and woody classes (Lawless, 1989). Stimuli were presented in $120-\mathrm{ml}$ screw-cap jars. Odorants were placed in the jars by dipping perfumer's blotter strips (Red Line, Frank Orlandi, Inc.) in the odorants to a depth of $4.5 \mathrm{~cm}$, placing the strips in the jars, capping the jars, and allowing the headspace to equilibrate. Jars were inspected by undoing the cap, sniffing the headspace, and replacing the cap.

The first group of subjects rated the "woody" and "citrus" character of dihydromyrcenol following the citrus conditioning set. The second group of subjects evaluated dihydromyrcenol following the woody conditioning set, on a different day. For both of these groups, the dihydromyrcenol sample was placed in the same testing booth as were the other samples; that is, no special attention was drawn to it. The order of the first five odorants-that is, the conditioning set-was randomized, and dihydromyrcenol was always rated last. Presentation order was controlled via random three-digit codes, which appeared on both the ballots and sample jars. For the third and fourth groups, the procedure was much the same, except that the dihydromyrcenol sample was evaluated in a different room, approximately $5 \mathrm{~m}$ from the first testing area. This was done to allow the sense of smell of the observers to recover from any potential olfactory adaptation. The third group smelled dihydromyrcenol after a citrus conditioning set and the fourth group after a woody conditioning set. Conditioning odors for the third and fourth groups were the same as for the first and second groups, respectively. All odors were 
rated on 9-point integer category scales for citrus and woody character. Scales were anchored with the words slightly at the low end and very at the high end-for example, "slightly woody" to "very woody."

\section{RESULTS}

In Figure 1, the citrus and woody ratings are shown for dihydromyrcenol for the four groups. A shift was seen as a function of the context within which citrus and woody character was rated for dihydromyrcenol. For the first two groups, the citrus character of dihyromyrcenol was enhanced in the context of predominantly woody odors, relative to citrus ratings made in a citrus context $[t(118)=$ $4.58, p<.001$ ]. Conversely, in a citrus context, woody ratings were enhanced relative to woody ratings made in a woody context $[t(118)=2.60, p<.05]$. Essentially the same pattern of results was seen for the third and fourth groups, with enhanced citrus ratings in the woody context $[t(108)=2.52, p<.05]$ and enhanced woody ratings in the citrus context $[t(108)=3.77, p<.001]$. To further assess the equivalence of the contextual shift under the two adaptation conditions, combined analyses of variance were performed with context and evaluation condition as factors. No significant interactions of context and condition were seen, confirming the similarity of the contextual shift in both conditions. That is, regardless of whether subjects evaluated dihydromyrcenol directly after smelling the conditioning odors, or whether they walked to another room, the shift was observed to be of about the same magnitude.

\section{DIHYROMYRCENOL RATINGS}

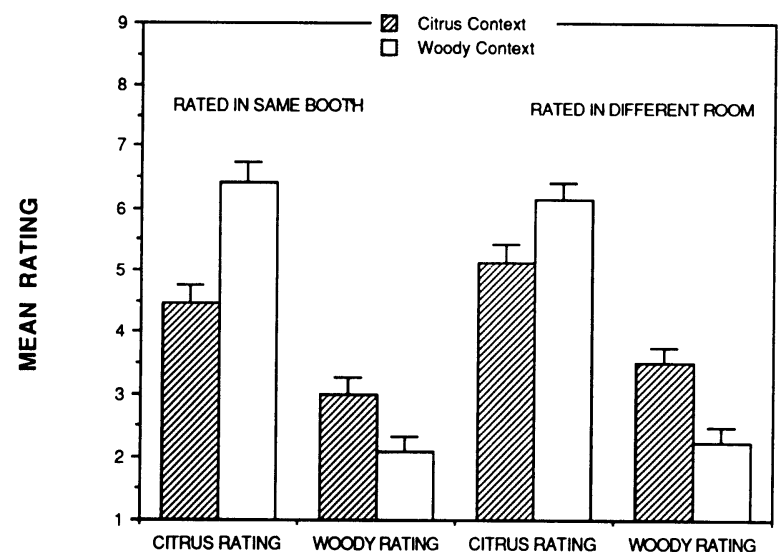

ODOR CHARACTERISTIC

Figure 1. Mean ratings (+1 SE) of dihydromyrcenol on scales for perceived intensity of citrus and woody character. Hatched bars show ratings when dihydromyrcenol was rated following five predominantly citrus-like odors. Open bars show ratings when dihydromyrcenol was rated following five predominantly woody odors. On the left side of the figure are ratings of dihydromyrcenol that were made in the same booth as were the five conditioning odors (immediately after them). On the right side of the figure are ratings for participants who walked to another room to evaluate dihydromyrcenol, to minimize potential effects of olfactory adaptation.
Table 1

Mean Ratings for Conditioning Odors

\begin{tabular}{llccc}
\hline \multicolumn{1}{c}{ Odorant } & Citrus & Woody & Citrus & Woody \\
\hline \multicolumn{5}{c}{ Citrus Set } \\
Limonene & 5.95 & 2.48 & 5.65 & 3.00 \\
Citral & 5.79 & 3.55 & 6.47 & 3.73 \\
Mandarin oil & 4.09 & 4.18 & 3.75 & 4.47 \\
Lemon oil & 5.84 & 3.30 & 5.18 & 4.22 \\
Orange oil & 7.00 & 2.29 & 6.49 & 2.51 \\
\multicolumn{5}{c}{ Woody Set } \\
Cedrene & 1.56 & 6.56 & 1.66 & \\
Sandalwood oil & 2.07 & 4.40 & 2.85 & 3.95 \\
Pine oil & 2.45 & 4.73 & 2.93 & 3.68 \\
Isobornyl acetate & 2.53 & 3.87 & 3.08 & 3.05 \\
Cedarwood oil & 2.00 & 6.40 & 1.66 & 5.78 \\
\hline
\end{tabular}

Note-The first and second columns contain ratings from the groups that rated the conditioning odors and dihydromyrcenol in the same room. The third and fourth columns contain ratings from the group that walked to a different room to rate dihydromyrcenol.

The mean citrus and woody ratings for the contextual stimuli that preceded the ratings of dihydromyrcenol are shown in Table 1 for each group. In all cases except that of mandarin oil, odorants in the a priori "citrus" group were rated higher in citrus character than they were rated in woody character, and all odorants in the a priori "woody" group were rated higher in woody character than they were rated in citrus character $(t$ tests, $p<.05)$.

\section{DISCUSSION}

This experiment provides the first psychophysical documentation of a sequential contrast effect on perceived odor quality. In general, two common theoretical explanations for context-dependent aftereffects involve either adaptation (fatigue) of specific neural elements or a shift in response criteria. In the latter view, repeated presentation of a good exemplar of a category makes the criteria for accepting another exemplar as a member of the category more stringent. Since both adaptation and criterion shifts produce effects that can be described as contrast effects, they are often difficult to differentiate as possible mechanisms (Samuel, 1986).

Simple sensory adaptation, defined as a decrease in perceived intensity following exposure to one type of odor, is still a potential candidate for the basis of this effect, since the control for adaptation-that is, walking to a separate room-did not include an assessment of the actual adaptation state of the subjects. One basis for adaptation-dependent shifts involves the fatigue of specific feature detectors that was once proposed as an explanation for apparent shifts in phoneme boundaries (Eimas \& Corbit, 1973; Samuel, 1986). Adaptation is also a potential mechanism if dihydromyrcenol produces sensations similar to an odor mixture in which two odor qualities are perceived simultaneously. Partial masking is usually observed between two odor qualities in mixtures (Cain, 1975; Lawless, 1986). After adaptation to one component of the mixture (reducing its perceived intensity), the other component often rebounds to a higher level-that is, it is released from masking effects (Lawless, 1987). Because of these potential mechanisms, the potential role of adaptation should be examined further.

Another explanation would involve a shift in response criterion. That is, the perception of the odorant might remain stable, but the cognitive boundary for classification as "citrus"' versus "woody" might shift as a function of exposure. It is not unreasonable to think that exposure to a set of highly prototypical odors for a given category might sharpen or shrink the momentary extent of that category, bringing boundaries in and causing borderline cases to then fall beyond the criteria or category limits. 
The effect could also arise from a change in response bias in the use of the rating scales, similar to Parducci's frequency principle (Parducci, 1965). That is, if subjects were uncomfortable making too many ratings in one section of the scale, they might welcome the opportunity with an ambiguous compound to make high ratings on a scale that had been overused on the low end, and to make low ratings on a scale that had previously been overused on the high end. This possibility could be addressed by having subjects make other ratings (pleasantness, overall intensity, etc.) or simply perform some other task during the contextual exposure phase of the procedure.

Whatever the mechanism or mechanisms underlying this effect, it appears to be a robust shift. Recent multidimensional scaling analyses have shown that "ambiguous" fragrance materials such as dihydromyrcenol form a cluster between true citrus (lemon, orange) odors and true woody (pine, cedar) odors in a perceptual map (Lawless, 1989). These boundary odors may behave like reversible figures-they may act like multistable Necker cubes in olfactory perception-in the sense that they will be perceived as woody under some circumstances and as citrus-like under others. Whether such shifts are characteristic of odors that fall on category boundaries or whether they are seen with more prototypical odors should be a subject for further study. The tendency to shift may be one objective criterion for differentiating prototypical from boundary odors in category structure.

\section{REFERENCES}

Blakemore, C., \& Sutton, P. (1969). Size adaptation: A new aftereffect. Science, 166, 246-247.
CaIN, W. S. (1975). Odor intensity: Mixtures and masking. Chemical Senses \& Flavor, 1, 339-352.

DiEHL, R. L. (1981). Feature detectors for speech: A critical reappraisal. Psychological Bulletin, 89, 1-18.

EImAS, P. D., \& CoRBIT, J. (1973). Selective adaptation of linguistic feature detectors. Cognitive Psychology, 4, 99-109.

Engen, T. (1982). The perception of odors. New York: Academic Press.

Koster, E. (1971). Adaptation and cross adaptation in olfaction. Unpublished doctoral dissertation, University of Utrecht.

LAWLESS, H. T. (1986). Sensory interactions in mixtures. Journal of Sensory Studies, 1, 259-274.

LAWLESS, H. T. (1987). An olfactory analogy to release from mixture suppression in taste. Bulletin of the Psychonomic Society, 25, 266-268.

LAWLESS, H. T. (1989). Exploration of fragrance categories and ambiguous odors using multidimensional scaling and cluster analysis. Chemical Senses, 14, 349-360.

MCBuRNEY, D. H., \& BARTOSHUK, L. M. (1973). Interactions between stimuli with different taste qualities. Physiology \& Behavior, 10, 1101-1106.

ParducCI, A. (1965). Category judgment: A range-frequency model. Psychological Review, 72, 407-418.

Samuel, A. G. (1986). Red herring detectors in speech perception: In defense of selective adaptation. Cognitive Psychology, 18, 452-499.

(Manuscript received January 7, 1991.) 